\title{
EL FÚTBOL COMO POTENCIADOR DE TURISMO DEPORTIVO
}

\author{
FOOTBALL AS A SPORTS TOURISM ENCOURAGEMENT
}

\author{
Rómulo Jacobo González-Garcia ${ }^{1}$ \\ Maria Huertas González-Serrano ${ }^{2}$ \\ David Parra-Camacho ${ }^{3}$
}

\section{Resumen}

El turismo deportivo se puede definir como un viaje de ocio con el fin de participar, de distintas formas, en actividades físico-deportivas. Respecto al fútbol, sus encuentros constituyen grandes eventos deportivos, atracciones donde el turista vivencia, principalmente de forma pasiva, distintas actividades, ya sea como espectador o como visitante de las atracciones turístico-futbolísticas. Sin duda, el fútbol tiene un importante valor turístico, en especial para la estructura turística local, incidiendo de manera directa en aspectos como la promoción y difusión de un destino turístico. En este marco, los clubs de fútbol se convierten en promotores turísticos de su ciudad, además de desarrollar otros recursos turísticos (museos, visitas, experiencias con jugadores, o paquetes para presenciar al equipo "fuera de casa"). El fútbol puede permitir configurar un producto turístico más atractivo, diversificado, que atrae nuevos nichos de mercado.

\section{Palabras clave}

Fútbol, turismo deportivo, eventos deportivos.

\section{Abstract}

Sports tourism can be defined as a leisure trip in order to participate, in different ways, in physical and sports activities. With regard to football, their meetings are major sporting events (social impact, broad level of public attendance, presence of media, television audience, ease of practice and high number of followers), attractions where tourists experience, mainly passively, different activities, either as a spectator or as a visitor of the tourist-soccer attractions. Undoubtedly, football has an important tourist value, especially for the local tourism structure, directly affecting aspects such as the promotion and dissemination of a tourist destination. In this context, football clubs become tourist promoters of their city, in addition to developing other tourist resources (museums, visits, experiences with players, or packages to witness the team "away from home"). Football can make it possible to create a more attractive, diversified tourism product that attracts new market niches.

\section{Key words}

Soccer, sports tourism, sporting events.

\footnotetext{
${ }^{1}$ Universidad de Valencia (España). Correo electrónico: rojagon@alumni.uv.es.

${ }_{3}^{2}$ Universidad de Valencia (España). Correo electrónico: m.huertas.gonzalez@uv.es.

${ }^{3}$ Universidad Católica de Valencia (España). Correo electrónico: david.parra@ucv.es
} 


\section{Fútbol y turismo deportivo}

La relación entre deporte y turismo ha estado estrechamente ligada, aumentando su importancia en las últimas décadas. De hecho, se puede observar que la historia muestra como las personas han relacionado el viajar con el deporte durante siglos. A modo de ejemplo, en la Antigua Grecia, los ciudadanos realizaban desplazamientos a otras ciudades con motivo de presenciar los acontecimientos en las olimpiadas (MacCannell, 2003). De este modo, con el transcurso de los años, el turismo deportivo se ha convertido en un nuevo campo académico de estudio, además de ser identificado el deporte como un producto turístico de manera cada vez más popular.

Asimismo, las ciencias sociales han destacado el potencial del fútbol como un espacio para la expresión de identidades colectivas (Bromberger, 2000) lo que se traduce en una fuerte presencia e influencia entre los hábitos deportivos y recreativos de la sociedad, siendo escasas las publicaciones que abordan este fenómeno desde un punto de vista sociológico, especialmente en el contexto español (Llopis-Goig, 2015).

Tal y como señala Jonker (2004), para conseguir un modelo turístico competitivo, los destinos deben seguir parámetros como una evaluación estratégica que represente un análisis global del sector (tanto nacional como internacional), donde se contemplen las tendencias turísticas actuales, así como los recursos y capacidades con las que cuenta el destino. De la misma manera, destaca el autor que la toma de decisiones estratégicas consta de dos partes: una primera donde se determina la dirección estrategia y el posicionamiento del destino y una segunda donde se identifique e integren los factores claves que lleven al éxito en materia turística.

Según los datos elaborados por KPMG Sports (2015), la influencia económica que posee este deporte en España es de alrededor de 7.600 millones de euros, lo que se traduce en un $0,75 \%$ del PIB (Producto Interno Bruto). Por otro lado, durante esta última temporada 2016/2017, los clubs y sociedades anónimas deportivas tuvieron contratados a 7.700 trabajadores, sumados a aquellos que son contratados por proveedores, subcontratistas y empresas de servicios, el número de trabajos a tiempo completo asciende a 75.300 empleados (Liga de Fútbol Profesional -LFP, 2016)

Sin embargo, cuando se habla sobre el impacto económico del fútbol en España, se hace referencia al fútbol profesional, concebido como espectáculo deportivo, por tanto, pensado como un producto de consumo masivo (Bahamonde, 2011). Indica Llopis-Goig (2015), que las tendencias o presiones ambientales que más influyen en la configuración y estructura de los clubs son las que implican los procesos de profesionalización, mercantilización y globalización. A continuación, se explican estos tres procesos:

1) La profesionalización responde a la búsqueda de un mayor desempeño en el desarrollo de las tareas y funciones, y suele significar que la dirección del club está en manos de técnicos y profesionales especializados. Es pues, una respuesta a la creciente complejidad del entorno y se manifiesta en una mayor diferenciación de funciones, planificación de acciones, coordinación entre las distintas áreas y búsqueda de mayor eficacia y eficiencia.

2) Los procesos de mercantilización de los clubs surgen históricamente cuando el incremento de los gastos internos obliga a buscar fuentes externas de financiación, lo que ha llevado a los clubs a crear y fortalecer sus departamentos comerciales y desarrollar estrategias de marketing, tanto con sus seguidores como con el público en general.

3) Por último, las tendencias de globalización han tenido un fuerte impacto en la configuración y los patrones de rendimiento de los principales clubs de fútbol. Este proceso afecta a la composición de los clubs, y está relacionado con la mundialización de las audiencias y la imparable formación de un complejo deportivomediático a nivel mundial.

\section{Turismo deportivo y turista deportivo}

En primer lugar, se debe definir qué se entiende por turismo deportivo y por turista deportivo. El turismo deportivo se puede definir como: un viaje de ocio por el que un individuo sale temporalmente de su lugar de residencia para participar en actividades físicodeportivas, para ver actividades físico-deportivas, o para visitar atracciones asociadas con actividades físicodeportivas. (Gibson, 1998). Este último, el turista, es identificado según Nogawa, Yamaguchi y Hagi (1996) como un visitante provisional, que se queda al menos 24 horas en la zona, y cuyo propósito principal es participar en un acontecimiento relacionado con el deporte, siendo la visita a la zona donde realiza la actividad un objetivo secundario.

En la actualidad, existen multitud de ofertas turístico- 
deportivas que varían a razón de lo exigido por el turista, adaptándose a las características e intereses de cada individuo. Si se profundiza en el turista deportivo, investigaciones previas sugieren que existen tres tipos distintos de comportamientos asociados al mismo:

1. Participante activo (Turismo de deporte activo),

2. Espectador (Turismo de eventos deportivos),

3. Visitante (Turismo deportivo de nostalgia).

Si se profundiza en los productos ofertados para el turista deportivo, Kurtzman (2005) realizó una descripción de la oferta de turístico-deportiva existente, lo que derivó en cinco productos diferenciados:

- Atracciones deportivas,

- Resorts deportivos,

- Tours deportivos,

- Cruceros deportivos,

- Eventos deportivos.

Según este mismo autor, además es esencial atender a una serie de factores a la hora de utilizar los recursos para realizar la actividad dentro de un entorno deportivo:

- Disponer de infraestructuras e interestructuras para los no residentes de la localidad.

- Proporcionar atractivos y servicios para los turistas.

- Planificar y comercializar con el fin de atraer a personas fuera de la localidad.

Por lo tanto, cabe señalar que estos factores se tendrían en cuenta al determinar la existencia de una "actividad turística deportiva”.

\section{Relación turístico - futbolística}

En materia económica, las dimensiones alcanzadas por el sector del fútbol están estrechamente relacionadas a la adopción por los principales clubs de modelos de gestión desde el mundo de los negocios (Llopis-Goig, 2015). Clubs como el Manchester United, Real Madrid o F.C Barcelona, contratan a profesionales de la industria del marketing. Asimismo, indica este autor, los clubs han extendido su gama de actividades con contratos de patrocinios de marcas mundiales, acuerdos de colaboración con clubs deportivos de otras áreas geográficas, acuerdos de difusión de contenidos audiovisuales a través de empresas del sector de las comunicaciones, así como el diseño y puesta en marcha de diversos proyectos relacionados con la responsabilidad social corporativa (Ginesta, 2011).
Concretando en materia futbolística, se destacan como atracciones deportivas la visita a museos, estadios, conferencias o congresos futbolísticos, etc. En cuanto a los resorts deportivos, existe una tendencia actual a proyectar hoteles relacionados a clubs de fútbol, o diseñar grandes espacios adaptados para que los equipos puedan realizar stages de pretemporada, preparaciones esporádicas en épocas invernales, etc. que pueden incidir en materia económica puntualmente en una localidad.

En cuanto a los tours, podemos diferenciar como en la actualidad, muchos clubs de fútbol punteros realizan durante el periodo de pretemporada giras con motivo de expansión y promoción internacional, pudiendo influir a su vez en la promoción turística de la localidad. Por último, los eventos deportivos, que abarcan desde los grandes campeonatos (Copa del Mundo, Eurocopa) hasta partidos a nivel provincial sin gran repercusión social.

Los procesos de internacionalización favorecen a los clubs en el aumento de sus seguidores en todo el mundo, lo que fomenta la participación en giras internacionales, sirviendo de esta manera como una interesante fuente de ingresos. De la misma manera, los clubs han desarrollado estrategias para el reparto de contenidos audiovisuales con empresas del mundo de la comunicación, siendo esta otra importante fuente de ingresos. (Llopis-Goig, 2015). Durante la última década, indica este autor, ha habido una gran brecha entre los clubs que han desarrollado una dimensión internacional en sus actividades y aquellos que, por el contrario, siguen vinculados a un escenario local.

\section{Eventos deportivos y futbol}

La confluencia de la administración del deporte y los estudios deportivos con el turismo se centran principalmente en dos temas importantes: los eventos deportivos como atracciones (para participantes y seguidores), y las formas más activas de participación deportiva que requieren realizar un viaje para poder realizarlas. Las agencias regionales de turismo generalmente incluyen eventos, atracciones y exposiciones como parte importante de su estrategia de desarrollo turístico (Getz, 1989).

Si esto lo relacionamos con el deporte rey de nuestra sociedad, dentro del ámbito turístico-futbolístico debemos descartar que el turista sea participante activo del deporte, puesto que en la mayoría de los casos éstos vivenciarán de forma pasiva la actividad, ya sea como espectador del evento o como visitante a alguna 
de las atracciones turístico-futbolísticas. La práctica activa en este caso, está reservada a los futbolistas, cuya profesionalización impide realizar actividades turísticas en la localidad, aunque su mera presencia puntual puede aumentar los beneficios económicos-turísticos de la localidad. De esta manera, podemos clasificar a la mayor parte de estos turistas futbolísticos como espectadores o visitantes.

Otro aspecto a tener en cuenta para que incide en la generación de turismo es la magnitud del evento. Se debe prestar atención a la magnitud que pueda presentar la celebración de tal práctica deportiva, ya que éste repercutirá con una mayor o menor influencia en el devenir económico-turístico de la localidad. Para ello, atendiendo a los eventos deportivos, para que una actividad deportiva pueda calificarse como tal, debe cumplir una serie de características que supongan un nivel de organización más elevado. Para Añó (2011) existen cuatro características que separan una actividad deportiva de un evento deportivo. Estas son las siguientes:

- Repercusión social,

- Nivel amplio de asistencia de público,

- Presencia de los medios de comunicación,

- Audiencia televisiva.

Estas condicionan la actividad física como evento deportivo, y a su vez están interrelacionadas entre sí, aunque en algunas situaciones específicas existan deportes que no tengan la suficiente repercusión en alguna característica concreta, pero poseen la suficiente solvencia como para categorizarlas como eventos deportivos. Siguiendo a este mismo autor, existen otras dos características que permiten diferenciar entre eventos deportivos y grandes eventos deportivos. Estas son:

- Tipo de deporte,

- Dificultad de la práctica.

Deportes como el fútbol cuentan con un número de seguidores y practicantes mucho mayor que cualquier otro tipo de deporte, frente a deportes cuya práctica es de un alto nivel técnico, que cuentan con un menor número de practicantes y en consecuencia, un menor seguimiento de las competiciones. Por último, el autor señala otras dos características fundamentales para considerar al evento deportivo como un mega-evento deportivo. Estas son:

- Patrocinadores,

- Ingresos propios privados (venta de entradas, derechos de retrasmisión, merchandising...).

Aunque puedan considerarse como una única característica, estas últimas se diferencian en que, en la actualidad, todos los grandes eventos deportivos cuentan con el apoyo de grandes casas comerciales para su celebración, mientras que los ingresos propios percibidos por los eventos deportivos varían enormemente según la práctica deportiva. Concretamente en el ámbito futbolístico, se cumplen las características definidas, puesto que posee una inmensa repercusión social, ya que suele asistir un mayor número de público comparado con otros deportes. Esto provoca una fuerte presencia en los medios de comunicación, los cuales consiguen altos porcentajes de audiencia televisiva en la mayoría de las ocasiones. Sin embargo, se debe comprender que el valor turístico del fútbol también depende, como se explica la Figura 1, de la magnitud y periodicidad de la competición.

Figura 1. Valor turístico de los eventos deportivos

\begin{tabular}{|c|c|c|}
\hline $\begin{array}{l}\text { Mega Eventos } \\
\text { Ocacionales }\end{array}$ & $\begin{array}{l}\text { Alta demanda turística; Alto valor } \\
\text { Ej. Mundial de Futbol, Eurocopa }\end{array}$ & Las características denominadas \\
\hline $\begin{array}{l}\text { Grandes Eventos } \\
\text { Periódicos }\end{array}$ & $\begin{array}{l}\text { Alta demanda turística; Alto valor } \\
\text { Ej. Champions League, Eurocopa League }\end{array}$ & $\begin{array}{l}\text { - Crecimiento potencial } \\
\text { - Cuota de mercado }\end{array}$ \\
\hline $\begin{array}{l}\text { Eventos } \\
\text { Regionales }\end{array}$ & $\begin{array}{l}\text { Media demanda turística; Medio valor } \\
\text { Ej. Liga Regular, Copa.. }\end{array}$ & $\begin{array}{l}\text { - Potenciación de la imagen } \\
\text { - Apoyo de la comunidad } \\
\text { - Valor medioambiental }\end{array}$ \\
\hline $\begin{array}{l}\text { Eventos } \\
\text { Locales }\end{array}$ & $\begin{array}{l}\text { Baja demanda turística; Bajo valor } \\
\text { Ej. Otras Competiciones }\end{array}$ & \\
\hline
\end{tabular}

Fuente: adaptado de Getz (2008). 
Con ello, los eventos ocasionales y periódicos (Copa del Mundo, Eurocopa...), aunque puedan generar grandes beneficios para las localidades de acogida, pueden generar costos económicos sustanciales, a menudo olvidados en la euforia que rodea el evento (Crompton, 1995). Sin embargo, si consideramos los eventos de menor escala (liga regular, copa...), éstos muestran un pequeño incremento en la llegada de turistas a la comunidad de acogida. Higham (1999) sugirió que los eventos deportivos a pequeña escala podrían dar como resultado unos efectos más positivos para las comunidades de acogida, por lo que la comunidad no debe desatender las competiciones deportivas de temporada regular.

Tabla 1. Diferencias entre mega eventos y competición regular.

\begin{tabular}{|c|c|c|}
\hline Aspecto & Mega eventos deportivos & Competición regular \\
\hline Proceso de licitación & $\begin{array}{l}\text { Principales costos asociados con el proceso } \\
\text { de licitación. Gasto público de licitación inflado } \\
\text { (en ocasiones a niveles agobiantes) por la } \\
\text { corrupción y patrocinadores intereses políticos. } \\
\text { La mejor oferta no es necesaria. El suministro } \\
\text { de intereses políticos y de patrocinadores } \\
\text { contribuye al éxito. }\end{array}$ & $\begin{array}{l}\text { Menores gastos incurridos durante el proceso } \\
\text { de licitación. En algunos casos, el proceso de } \\
\text { licitación no se requiere en absoluto (por ejemplo, } \\
\text { competiciones deportivas de temporada regular). } \\
\text { La oferta más adecuada suele tener éxito. }\end{array}$ \\
\hline Aspectos de desarrollo & $\begin{array}{l}\text { Costos de desarrollo significativos asociados } \\
\text { con eventos deportivos como los Juegos } \\
\text { Olímpicos y el Mundial de fútbol. Beneficios } \\
\text { económicos asociados con los desarrollos } \\
\text { de infraestructura recibidos por intereses } \\
\text { empresariales y no por la comunidad de } \\
\text { acogida. }\end{array}$ & $\begin{array}{l}\text { Generalmente existe infraestructura. Se realiza } \\
\text { dentro de los umbrales de capacidad de la ciudad } \\
\text { anfitriona. Los costes de los desarrollos de } \\
\text { infraestructuras suelen ser adecuados a la escala } \\
\text { de la ciudad anfitriona. }\end{array}$ \\
\hline Desarrollo de legado & $\begin{array}{l}\text { Legado de instalaciones infrautilizadas y caras } \\
\text { con deuda financiera asociada. }\end{array}$ & $\begin{array}{l}\text { La mejora de las instalaciones (si es necesario) } \\
\text { beneficia a los deportistas, espectadores y } \\
\text { administradores. }\end{array}$ \\
\hline Beneficios económicos & $\begin{array}{l}\text { Dominado por grandes empresas y } \\
\text { patrocinadores. Los residentes locales ven } \\
\text { relativamente poco beneficio económico. } \\
\text { Medios efectivos para obtener dinero del erario } \\
\text { público y reubicarla en intereses privados. }\end{array}$ & $\begin{array}{l}\text { Comunidad local más propensos a participar en } \\
\text { los beneficios económicos positivos asociados con } \\
\text { el deporte. Mucho menos carga sobre los fondos } \\
\text { públicos. }\end{array}$ \\
\hline $\begin{array}{l}\text { Beneficios turísticos a } \\
\text { corto plazo }\end{array}$ & $\begin{array}{l}\text { Aumento a corto plazo del turismo compensado } \\
\text { por el cambio de horario. Desplazamiento de } \\
\text { turistas comúnmente asociados con mega } \\
\text { eventos deportivos. Los "adictos al deporte" } \\
\text { demuestran poco interés en probar el producto } \\
\text { turístico más amplio en el destino. }\end{array}$ & $\begin{array}{l}\text { Es probable que los visitantes sean viajeros } \\
\text { más frecuentes que temporales. Ocurren menos } \\
\text { desplazamientos de turistas si los deportes } \\
\text { complementan la escala de la infraestructura de } \\
\text { la ciudad anfitriona. Los turistas probablemente } \\
\text { experimenten un producto turístico más amplio del } \\
\text { destino. }\end{array}$ \\
\hline $\begin{array}{l}\text { Beneficios turísticos a } \\
\text { medio plazo }\end{array}$ & $\begin{array}{l}\text { Retraso a mediano plazo en el turismo de larga } \\
\text { distancia asociado a mega-eventos debido al } \\
\text { cambio de horario. }\end{array}$ & $\begin{array}{l}\text { Es poco probable que los patrones turísticos a } \\
\text { medio plazo se vean influidos por el cambio de } \\
\text { tiempo. }\end{array}$ \\
\hline Imagen de destino & $\begin{array}{l}\text { Mucho que perder debido a la mala publicidad, } \\
\text { las limitaciones de capacidad, los costos } \\
\text { financieros, el activismo político y el terrorismo. }\end{array}$ & $\begin{array}{l}\text { La imagen de destino que está en juego no es tan } \\
\text { alta. Gran potencial para que el turismo deportivo } \\
\text { actúe como vehículo promocional si se reconocen } \\
\text { las oportunidades. }\end{array}$ \\
\hline Aspectos sociales & $\begin{array}{l}\text { La aglomeración y la congestión de la } \\
\text { infraestructura turística a menudo asociada } \\
\text { con mega-eventos. Los residentes locales } \\
\text { a menudo se excluyen de la participación } \\
\text { en el evento debido al costo. Estilos de vida } \\
\text { locales generalmente interrumpidos por mega- } \\
\text { eventos y problemas de seguridad. }\end{array}$ & $\begin{array}{l}\text { La aglomeración y la congestión infraestructural } \\
\text { son menos probables si la escala de la ocasión } \\
\text { es apropiada para la ciudad anfitriona. Mayor } \\
\text { potencial de participación de los residentes locales } \\
\text { en la ocasión deportiva. }\end{array}$ \\
\hline
\end{tabular}




\begin{tabular}{|c|l|l|}
\hline $\begin{array}{c}\text { Problemas con } \\
\text { residentes locales: }\end{array}$ & $\begin{array}{l}\text { El desplazamiento o la expulsión de } \\
\text { residentes locales ocurre donde las ciudades } \\
\text { están ansiosas de capitalizar la imagen de } \\
\text { destino. Instalaciones desarrolladas a menudo } \\
\text { en áreas socioeconómicas más bajas. } \\
\text { Desplazamientos de la comunidad de acogida, } \\
\text { desalojos, aumentos de tarifas y alquileres. }\end{array}$ & $\begin{array}{l}\text { Impacto insignificante en los residentes locales. } \\
\text { Impacto positivo en aquellos que deciden participar. } \\
\text { Mayor de acceso local a las ocasiones } \\
\text { deportivas. }\end{array}$ \\
\hline Cuestiones políticas & $\begin{array}{l}\text { Posible secuestro del deporte como vehículo } \\
\text { político. }\end{array}$ & $\begin{array}{l}\text { Falta de escala e importancia para ser utilizado } \\
\text { como un vehículo político. }\end{array}$ \\
\hline Aspectos de seguridad & $\begin{array}{l}\text { Costes de seguridad significativos y riesgos } \\
\text { asociados con mega-eventos deportivos. }\end{array}$ & $\begin{array}{l}\text { Problemas de seguridad insignificantes y costos } \\
\text { financieros asociados con ocasiones deportivas. }\end{array}$ \\
\hline
\end{tabular}

Fuente: adaptado de Higham (1999)

Como se observa en la tabla 1, existe una enorme diferencia entre los aspectos asociados a los mega-eventos deportivos frente a los de pequeña escala. En estos niveles del deporte, el potencial de impacto negativo es mínimo. Puntualmente, en materia futbolística, debido a su gran repercusión mediática, pueden existir momentos determinados de la temporada regular que de pie a un gran acontecimiento deportivo, con la movilización a todos los niveles de un gran evento, es el caso de la celebración de los denominados "derbis", cuyo máximo exponente se encuentra en los partidos disputados entre Real Madrid y Barcelona y viceversa en la Liga Española. Por lo tanto, el nivel deportivo de los equipos que disputan los partidos, y por ello la incertidumbre del resultado, también es un factor influyente.

\section{Impactos asociados a la actividad turístico - futbolística}

En cuanto a los impactos asociados a la celebración de los acontecimientos deportivos, inclusive el fútbol, pueden catalogarse en: económicos, turísticos-comerciales, físicos-ambientales, socioculturales, psicológicos y político-administrativo. Centrándonos en los impactos turístico-comerciales y económicos del fútbol, la tabla 2 muestra una serie de impactos, tanto positivos como negativos asociados a la celebración de estos acontecimientos.

Tabla 2. Impactos positivos y negativos económicos y turístico-comercial de los eventos deportivos.

\begin{tabular}{|c|c|c|}
\hline Tipo de Impacto & Economía & Turismo / comercial \\
\hline Positivo & $\begin{array}{l}\text { - Incremento de la actividad económica. } \\
\text { - } \quad \text { Increación de empleo. } \\
\text { - Incremento de las ofertas de trabajo. } \\
\text { - Incl nivel de vida. }\end{array}$ & $\begin{array}{l}\text { - Aumento de la concienciación de la ciudad como desti- } \\
\text { no turístico. } \\
\text { Mayor conocimiento sobre el potencial para la inversión } \\
\text { y el desarrollo de la actividad comercial en la región. } \\
\text { - Creación de nuevos alojamientos y atracciones para los } \\
\text { turistas. }\end{array}$ \\
\hline Negativo & $\begin{array}{l}\text { - Incremento de los precios durante el evento. } \\
\text { - Especulación inmobiliaria. } \\
\text { - } \text { Falta de atracción de turistas. } \\
\text { - } \text { Mejores alternativas para las inversiones. } \\
\text { Capital inadecuado y estimación inadecuada de los } \\
\text { - Gastes del evento. } \\
\text { - Excesivo endeudamiento } \\
\text { - } \text { Aumento de los impuestos }\end{array}$ & $\begin{array}{l}\text { Adquisición de una mala reputación como resultado } \\
\text { de instalaciones inadecuadas, delincuencia, prácticas } \\
\text { impropias o precios inflados. } \\
\text { Reacciones negativas de las empresas existentes debi- } \\
\text { do a la posibilidad de nueva competencia de mano de } \\
\text { obra local y de la ayuda del gobierno }\end{array}$ \\
\hline
\end{tabular}


Según el estudio realizado por Llopis-Goig (2013) sobre las pautas de compromiso y vinculación de los seguidores de un equipo de futbol en España, indica que el comportamiento más común entre los seguidores es el consumo de partidos televisados (72.8\%), de los cuales dos de cada diez espectadores paga para ver partidos de su equipo en televisión (21\%), frente al 36,9\% de los ciudadanos que asisten a partidos de su equipo en directo. y la asistencia en el estadio. Menos común es ir a otra ciudad o país a seguir al equipo, que se realiza sólo $15.3 \%$ y $2.6 \%$ respectivamente.

Según datos de la Liga de Fútbol Profesional (LFP), durante la temporada 2015-16 el número de personas que asistieron a un partido de fútbol en un estadio ascendió a 14 millones. (LFP, 2016).

Entre una gama de variables que determinan el consumo de los asistentes en un evento deportivo, Preuss y Schütte (2009) identifican cuatro:

- El origen del asistente,

- El hecho de si el asistente realiza visitas a la localidad o únicamente al estadio,

- El hecho de si el asistente realiza pernoctaciones o no,

- La intención del asistente para visitar el evento.

Respecto a los motivos de los turistas para la asistencia a los eventos deportivos suelen ser muy variados. De hecho, Funk, Mahony, Nakazawa y Hirakawa (2001) identificaron diez dimensiones para predecir la asistencia a eventos deportivos: ser fanático del deporte, logros vicarios, entusiasmo, identificación de equipos, apoyar las oportunidades de las mujeres en el deporte, la estética, la socialización, el orgullo nacional, el drama y el interés en jugadores particulares.

Concretamente en fútbol, durante la temporada regular, el motivo de asistencia principal a los estadios podemos reducirla a la identificación con los equipos o el interés en jugadores particulares. Si atendemos a grandes eventos como el Mundial o la Eurocopa, pueden aparecer otros agentes como, el orgullo nacional o la socialización como motivo de asistencia al partido. Zhang et al. (2001) sostienen que la motivación es una base insuficiente para comprender los factores que impulsan la asistencia a eventos deportivos. Sin embargo, estos autores encontraron que las características demográficas de los espectadores (por ejemplo, edad, género, nivel educativo) se suman a la predicción de la frecuencia de asistencia que se logró utilizando únicamente las dimensiones motivacionales.

Otro aspecto a destacar es que la satisfacción de los turistas con el evento es importante para la asistencia, la imagen de destino, y la percepción sobre esta, y la intención de repetir la experiencia (Kaplanidou y Gibson, 2010). Higham y Hinch (2001) encontraron que el desarrollo de una imagen de destino entre los turistas parecía estar relacionado con la exposición de la región recibida viendo los partidos, así como de la cobertura que los medios hacían sobre los equipos. Aunque también es importante reconocer el papel del club de fútbol como promotor turístico de la ciudad. Aquellos clubs con mayor renombre internacional, suelen tener entre sus espectadores a un gran número de turistas extranjeros.

Por otra parte, cabe destacar que en los últimos tiempos se ha observado como los clubs de fútbol han comenzado a aplicar otro tipo de recursos para la atracción de turistas aparte de los eventos deportivos. Destacan entre ellos la aparición de los museos para presenciar los logros de los clubs. En algunos casos, estos incluyen recorridos por los estadios e incluso, alguna experiencia con jugadores históricos del club. Por ejemplo, el Tour del Bernabéu (Real Madrid), es el $5^{\circ}$ museo más visitado de España y al igual que el Camp Nou Experience ( F.C. Barcelona ) entre otros, ofrece una visita guiada por la colección de trofeos del club, así como un tour que recorre las dependencias del estadio, como vestuarios, palco... Destaca también el Old Trafford Museum (Manchester United), cuya visita puede estar guida por alguna de las figuras históricas del Club.

En la actualidad, la mayor parte de los clubs ofrecen paquetes de viaje que incluyen la entrada para presenciar a su equipo "fuera de casa". Irwin y Sandler (1998) indagaron en la planificación de los viajes y los patrones de gastos de los aficionados que asistieron a estos eventos, encontrando que los fans gastaron la mayor parte de su dinero en el alojamiento y las compras cotidianas, y que pasaban más tiempo en el destino que el del mero evento deportivo.

\section{Conclusión}

En definitiva, se puede utilizar el deporte para hacer el producto turístico más atractivo. Al promocionar y desarrollar un producto más diversificado, se amplían las posibilidades de atraer nuevos nichos de mercado. Fruto de ello ha sido la evolución observada dentro del ámbito futbolístico, en el que además de los eventos éstos 
han desarrollado otro tipo de estrategias como son los recorridos guiados por los estadios y museos deportivos. Esto exige una planificación previa, y en esa planificación se debe contar con la opinión de los residentes del destino turístico sobre sus percepciones del impacto económico, social y medioambiental del turismo. Concretamente, el fútbol como deporte de masas, posee todas las características que le permiten ser un valor importante dentro de la estructura turística local, incidiendo de manera directa en aspectos como la promoción, difusión y valor de un destino turístico.

\section{Referencias}

1. Añó, V. (2011). La Organización de Eventos y Competiciones Deportivas. Valencia: Universidad de Valencia.

2. Crompton, J.L. (1995). Economic Impact Analysis of Sports Facilities and Events: Eleven Sources of Misapplication. Journal of Sports Management, 9(1), $14-35$.

3. Fourie, J., \& Spronk, K. (2011). South African mega-sport events and their impact on tourism. Journal of Sport and Tourism, 16(1), 75-97. doi: 10.1080/14775085.2011.576119.

4. Funk, D. C., Mahony, D. F., Nakazawa, M., \& Hirakawa, S. (2001). Development of the sport interest inventory (SII): Implications for measuring unique consumer motives at team sporting events. International Journal of Sports Marketing and Sponsorship, 3(3), 291-316. doi: 10.1108/IJSMS-0303-2001-B005

5. Getz, D. (1989). Special Events: Defining the product. Tourism Management, 10(2), 125-131. doi: 10.1016/0261-5177(89)90053-8.

6. Getz, D. (2008). Event tourism: Definition, evolution, and research. Tourism Management, 29(3), 403-428. doi: 10.1016/j.tourman.2007.07.017

7. Gibson, H. J. (1998). Sport tourism: A critical analysis of research. Sport Management Review, 1(1), 45-76. doi: 10.1016/S1441-3523(98)70099-3

8. Gibson, H. J., Willming, C., \& Holdnak, A. (2003). Small-scale event sport tourism: fans as tourists. Tourism Management, 24(2), 181-190. doi: 10.1016/ s0261-5177(02)00058-4

9. Higham, J. (1999). Commentary-sport as an avenue of tourism development: An analysis of the positive and negative impacts of sport tourism. Current Issues in Tourism, 2(1), 82-90. doi: doi. org/10.1080/13683509908667845

10. Higham, J., \& Hinch, T. (2001). Sport and development at tourism destinations: Exploring mutually beneficial links. Paper presented at the Leisure Studies Association Conference (Journeys in Leisure: Current and Future Alliances), University of Luton, July 17-19.

11. Irwin, R., \& Sandler, M. (1998). An analysis of travel behaviour and event-induced expenditures among American collegiate championship patron groups. Journal of Vacation Marketing, 4(1), 78-90. doi: 10.1177/135676679800400107

12. Jonker, J. A. (2004). The strategic identification and integration of critical success factors to achieve international competitiveness for South Africa as a tourism destination. Tesis Doctoral, Universidad de Pretoria, Sudáfrica.

13. Kaplanidou, K \& Gibson, H. J. (2010). Predicting Behavioural Intentions of Active Event Sport Tourists: The Case of a Small-scale Recurring Sports Event, Journal of Sport \& Tourism, 15(2), 163-179, doi: 10.1080/14775085.2010.498261

14. KPMG Sports (2015). Impacto socio-económico del fútbol profesional en España. Suiza: KPMG International.

15. Kurtzman, J. (2005). Sports tourism categories. Journal of Sport \& Tourism, 10(1), 15-20. doi: 10.1080/14775080500101502

16. Liga de Fútbol Profesional (2016). Informe económico-financiero del fútbol profesional. Madrid: Liga de Fútbol Profesional.

17. MacCannell, D. (2003). El turista. Una nueva teoría de la clase ociosa. Barcelona: Melusina.

18. Nogawa, H., Yamaguchi, Y., \& Hagi, Y. (1996). An empirical research study on Japanese sport tourism in sport-for-all events: Case studies of a single-night event and a multiple-night event. Journal of Travel Research, 35(2), 46-54. doi: 
$10.1177 / 004728759603500208$

19. Preuss, H., \& Schutte, N. (2008). Football tourists and their contribution to the economic impact-evidence from EURO 2008 in Austria/Switzerland. European Association for Sport Management Conference, Heidelberg. Germany.

20. Preuss, H., y Solberg, H. (2006). Attracting major sporting events: The role of local residents. European Sport Management Quarterly, 6(4), 391-411. doi:

\section{$10.1080 / 16184740601154524$}

21. Llopis-Goig, R. (2015). Spanish Football and Social Change. Palgrave Macmillan, UK.

22. Zhang, J. J., Pease, D. C., Lam, E. T. C., Bellerive, L. M., Pham, U. L., Williamson, D. P., Lee, J. T., \& Wall, K. A. (2001). Sociomotivational factors affecting spectator attendance at minor league hockey games. Sport Marketing Quarterly, 10(1), 43-56. 